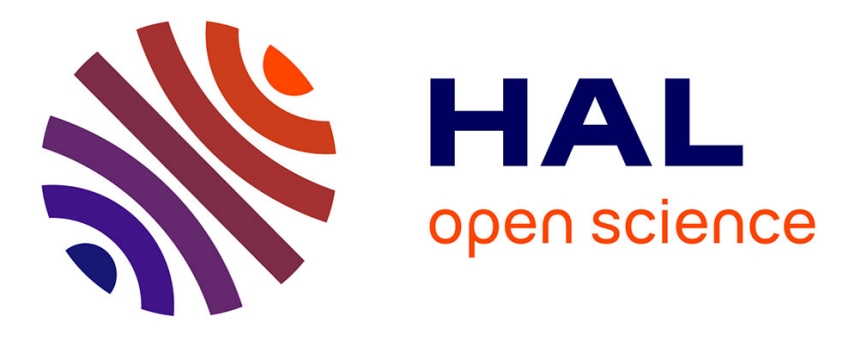

\title{
Simulation of Endoscopic Surgery
}

Nicholas Ayache, Stéphane Cotin, Hervé Delingette, Jean-Marie Clément, Jacques Marescaux

\section{To cite this version:}

Nicholas Ayache, Stéphane Cotin, Hervé Delingette, Jean-Marie Clément, Jacques Marescaux. Simulation of Endoscopic Surgery. Minimally Invasive Therapy and Allied Technologies, 1998, 7 (2), pp.71-77. inria-00615086

\section{HAL Id: inria-00615086 \\ https://hal.inria.fr/inria-00615086}

Submitted on 17 Aug 2011

HAL is a multi-disciplinary open access archive for the deposit and dissemination of scientific research documents, whether they are published or not. The documents may come from teaching and research institutions in France or abroad, or from public or private research centers.
L'archive ouverte pluridisciplinaire HAL, est destinée au dépôt et à la diffusion de documents scientifiques de niveau recherche, publiés ou non, émanant des établissements d'enseignement et de recherche français ou étrangers, des laboratoires publics ou privés. 


\title{
Simulation of \\ Endoscopic Surgery
}

\author{
Nicholas AYACHE ${ }^{l}$ - Stéphane COTIN ${ }^{l}$ - Hervé DELINGETTE ${ }^{1}$ \\ Jean-Marie CLEMENT ${ }^{2}$ - Jacques MARESCAUX ${ }^{2}$
}

\author{
${ }^{1}$ Projet EPIDAURE \\ INRIA Sophia Antipolis
}

2004, route des Lucioles - BP 93

06902 Sophia Antipolis Cedex

France

\author{
2 IRCAD \\ Hôpitaux de Strasbourg \\ 1, Place de l' hôpital \\ 67091 Strasbourg Cedex \\ France
}

\author{
tel : $(+33) 0493657663$ \\ fax : (+33) 0493657669 \\ e-mail : Nicholas.Ayache@ sophia.inria.fr \\ http://www.inria.fr/epidaure/
}

\section{Introduction}

Medical images contain a lot of information on the internal geometry and on the functions of the human body. The introduction of local storage capacities in each production site, and the introduction of telematic links between production sites and surgical departments with high bandwidth networks have open new possibilities in the preparation, control and validation of therapy. This is particularly true for video-surgery, where the use of video-images during the intervention makes the use of medical and virtual images during training or rehearsal sessions quite natural.

The precise geometrical and physical information given by medical images can be used to create a model of an average virtual patient, in order to train the surgeon on different types of surgical procedures. Also, pre-operative images of a specific patient can be used to create virtual « copy » of a particular case, in order to rehearse a difficult surgical procedure. In the future, we believe that surgery simulators should become as common for surgeons as flight simulators for pilots.

In this article, we describe the virtual environment we have created in order to develop a simulator of liver surgery including visual and haptic feedback: the surgeon can interact in real time with a image of the virtual organ, and both look and feel the deformations. This work was done in 
the Epidaure project at INRIA Sophia-Antipolis (France) in collaboration with IRCAD in Strasbourg (France). Epidaure is a research group specialized in medical images analysis, and IRCAD is a multidisciplinary research institute for cancers of the digestive tract, involving among others clinicians, surgeons and scientists.

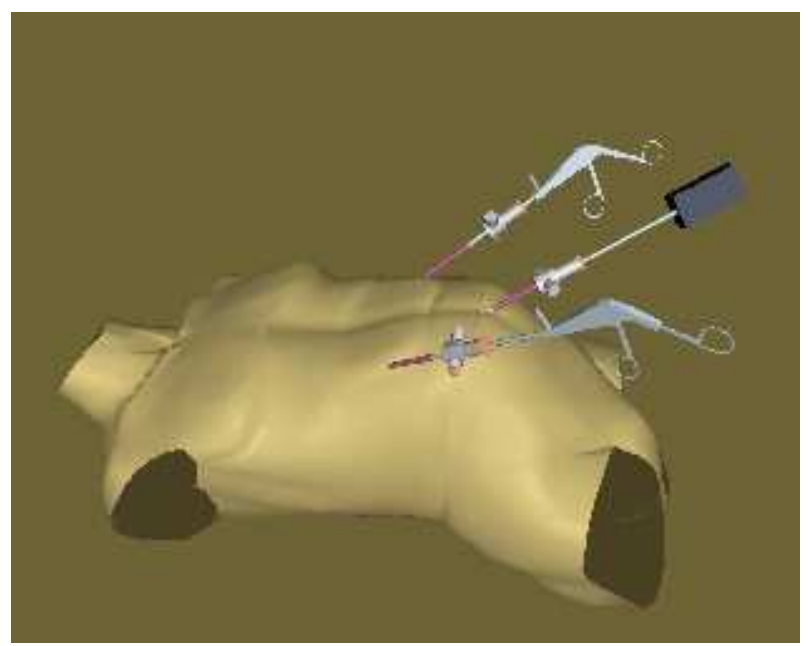

Figure 1: Minimally Invasive Surgery principle. The surgeon can see the operative field using a micro video camera. Consequently, the manipulation of the surgical tools is more complex than conventional open surgery.

Origin : Forschungszentrum Karlruhe

\section{Laparoscopic Surgery}

Laparoscopic surgery belongs to a set of techniques called Minimally Invasive Therapy, where the intervention in the abdomen is made through small incisions with elongated instruments. Images of the operation field are acquired thanks to an optical fiber also introduced through a small incision and linked to a mini-camera, and are displayed on a TV-screen placed above the patient (cf. figure 1).

This approach has many advantages for the patient: small incisions, reduced operation traumatises, and post-operative morbidity. Also the hospital period of stay can be drastically reduced. On the other hand, this technique requires new capabilities from the surgeon. First, degrees of freedom of the instruments are reduced, therefore introduction in the abdomen has to go through a fixed point (troccard). Second, the vision of the operation field is distorted by wide angle lenses, and does not correspond to an observation from the surgeon's point of view. In addition, the location of the TV-screen is not in the direction of the instruments. This requires a particular hand-eye coordination.

\section{Simulation of hepatic surgery}

Therefore a surgeon must practice thoroughly this new technique with the new sensations attached to it. Training on cadavers or animals raises a number of practical and ethical problems. Mechanical devices are extremely limited and do not offer a realistic simulation. The advantages of a computer simulator are numerous. Different organs and pathologies can be represented within a common framework, in order to train the surgeon to a large number of situations. Also, they can be adapted to the case of a specific patient. A number of groups (TIMC - Grenoble University Hospital LIFL - University of Lille - France, KISMET - Karlsruhe - Germany, HT - Washington and MERL - 
Boston - USA) regrouping computer scientists and physicians are working towards the development of surgery simulators. The collaboration between INRIA and IRCAD has at two goals: first the creation of an anatomical model of the liver to teach anatomy; second, the development of a surgery simulator to teach medical gestures.

\section{Geometrical modelling}

In order to create a very realistic model of the liver, we used the images of the Visible Man, distributed by the National Library of Medicine (USA). These images are high resolution (1 mm) colour pictures of cryogenic cross-sections of a cadaver. We used the digital image processing tools of the Epidaure group to extract the external boundary of the liver, and also the various vessels (veina cava, portal vein, hepatic artery, billiary duct). Then a volumetric representation is created ().

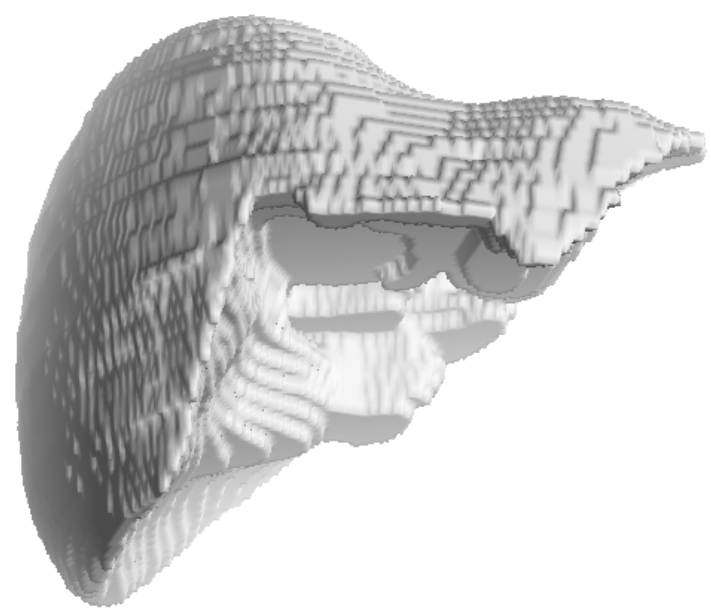

Figure 2:. liver model built from a set of $2 D$ segmented medical images.

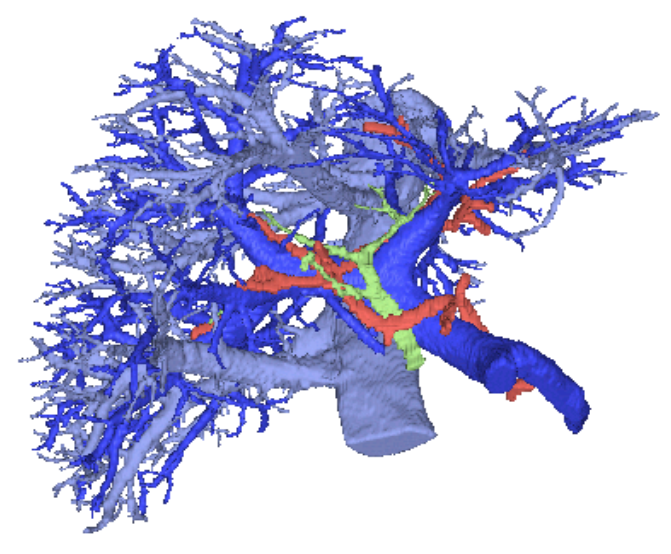

Figure 3: result of the vascular tree segmentation. The high quality of the original images allows a high level of detail in the reconstructed model.

Finally, an isosurface extraction [1] provides the mesh of a 3-D surface corresponding to each structure (cf. figure 3). One obtains a set of 5 surface meshes, either triangulated or with a simplex mesh [2] [3]. These surfaces can be manipulated interactively through a graphical interface. The surgeon can therefore plan surgery with the accurate knowledge of vessels location with respect to the liver external boundary, which is the only visible item during the actual surgery. Thanks to this 3D reconstruction, it is possible to visualise with transparency the different vessels of the liver, and therefore to plan the impact of each medical gesture. Doing this can reduce drastically the risk of cutting a major vein or artery during surgery. 


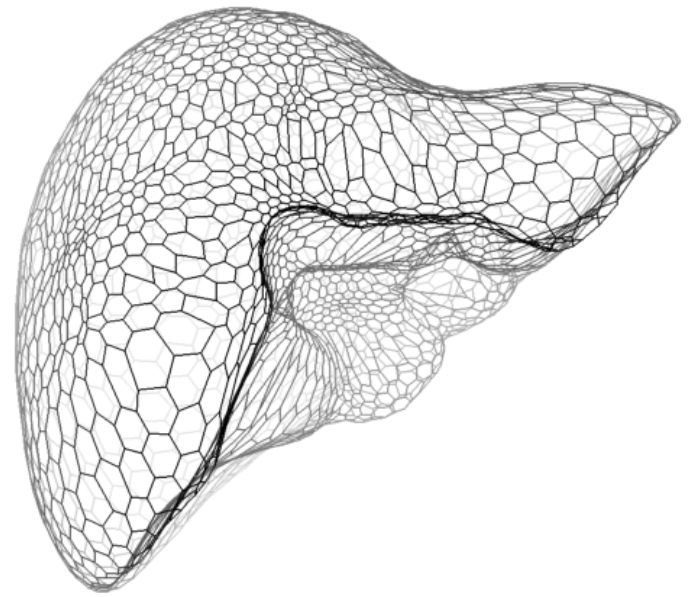

Figure 4: surface mesh of the liver. A minimisation of the number of vertices - without a lack of accuracy - is possible with the use of an adaptive simplex mesh.

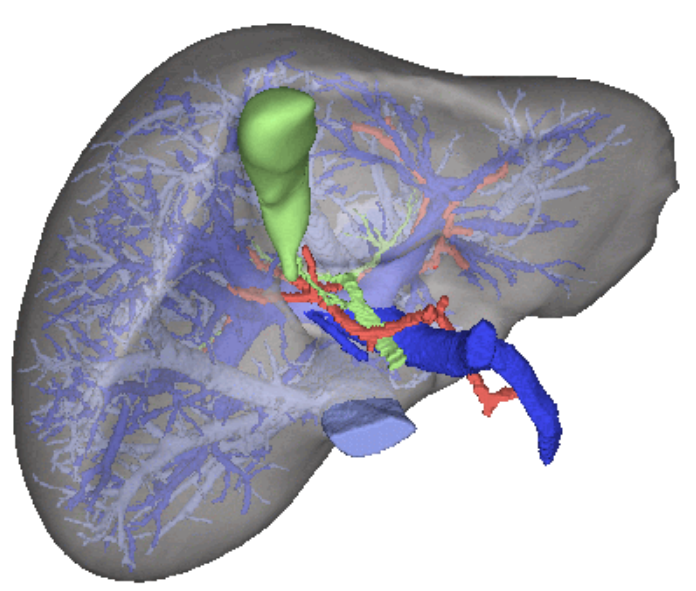

Figure 5: anatomical liver model. The visualisation of the vascular tree is possible with the use of transparency properties. It is possible to manipulate the $3 D$ mesh in real-time.

\section{Physical modelling}

The creation of an accurate model of the liver is only one step for surgery simulation. In effect, as said before, a major goal is to render the sensations linked to laparascopy, in order to better practice the required hand-eye coordination. For visual realism, we must simulate what appear on the video screen. This implies that it is not necessary to be «immersed» in a virtual world for this kind of simulation. On the other hand, the tactile feed-back is an essential complement to the visual feed-back, and we believe that an efficient simulator must be equipped with force feedback.

Organs are deformable, and their deformation laws are in general complex and unknown. A number of approaches have been proposed to model deformable objects in graphics $[4,5,6,7]$, or in a domain closer to physiology [8] [9]. We have chosen a relatively simple model, but with a lot of advantages including speed. Our objective is to develop a simulator which does not remain a laboratory prototype, but could be industrialised for surgeons benefit. To achieve this goal, the chosen material must be standard and of reasonable cost.

Our chosen model should allow:

- real time deformations $(25 \mathrm{~Hz}$ for display, between $300 \mathrm{~Hz}$ and $500 \mathrm{~Hz}$ for force feedback).

- volumetric modeling of organs, allowing realistic deformations and simulation of cutting, suturing, etc.

- modelling anisotropic linear elasticity properties of liver tissues and vessels (although their is a lack of biomechanical data available on this topic).

\section{Force feedback}

Recent work has shown that the notion of presence in a virtual environment is not only dependant on visual realism but greatly enhanced by other perception means $[10,11]$. In particular, the sensation of forces and textures is very important in medical applications [12, 13]. Force feedback remains essential to simulate realistically the technique. This is the only way to restore the «physical » contact between the operator and virtual objects. 
We chose to use the force feedback system called Laparoscopic Impulse Engine ${ }^{\mathrm{TM}}$. This system has many attractive properties including 5 degrees of freedom ( 3 of them being motor controlled), an accuracy in displacement of about $0.025 \mathrm{~mm}$ and maximum intensity force in the direction of the trocard of $8.9 \mathrm{~N}$. This system is integrated in an architecture with 2 workstations linked with ethernet (cf. figure 6 and figure 11) .

To integrate such a system with our simulator, it is necessary to determine which forces are implied. To respect the constraints of speed and realism, linear elasticity theory appears rather attractive. This theory allows a better physical modelling than mass-spring systems, in particular because the reacting force is linked to the object deformation and to its elastic properties by the continuum mechanics relations. Linear elasticity has often been used [13, 14, 15] as a good approximation - for small displacements - of a deformable body behaviour, but the resolution of the attached equations requires finite element techniques which are quite costly in terms of CPU. To reach real time performances, we have introduced specific pre-computations of elementary deformations.
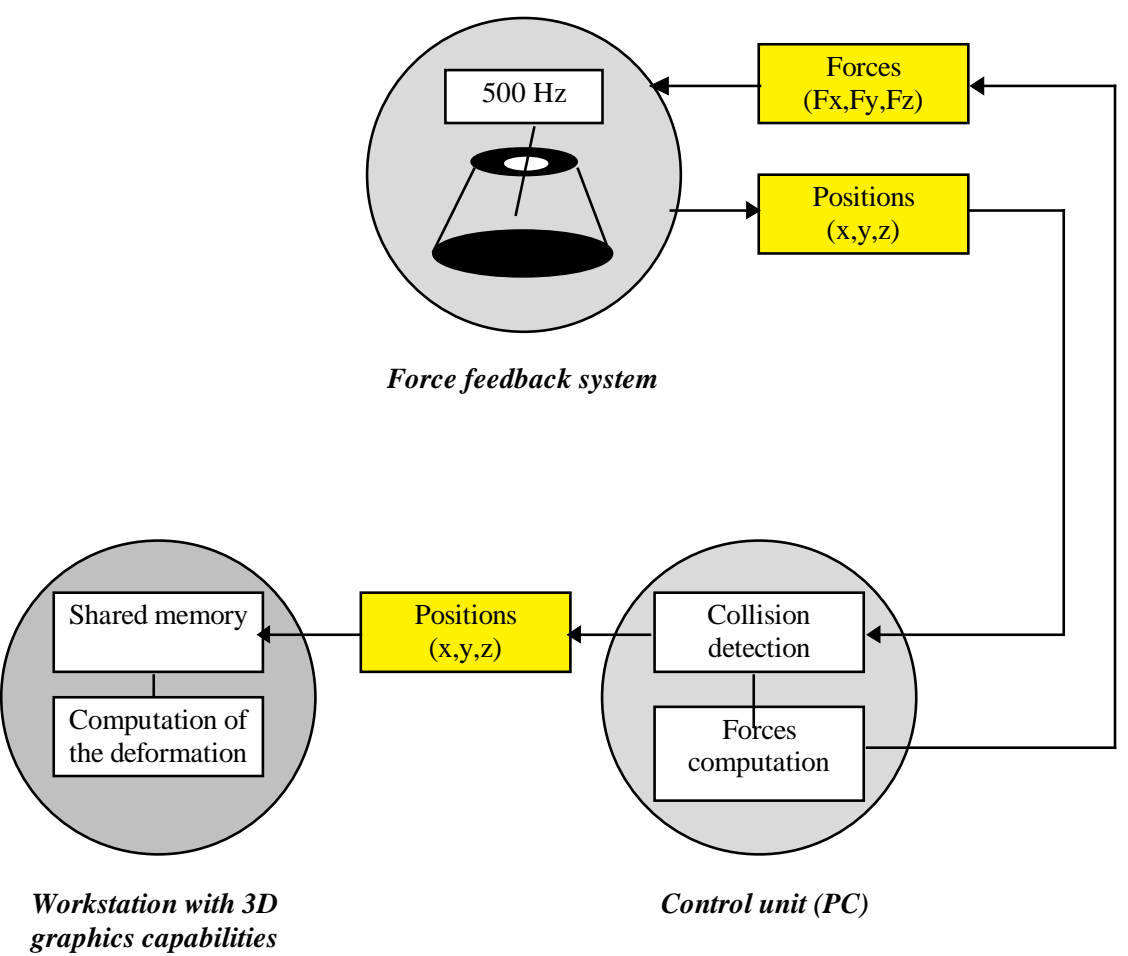

Figure 6: the actual hepatic surgery simulator prototype includes a workstation with $3 D$ graphics capabilities, a control unit (PC Pentium) and a force feedback system «Laparoscopic Impulse Engine ».

\section{$3 D$ linear elasticity}

Let us recall briefly the bases of linear elasticity. Let $\Omega_{0}$ be the configuration of an elastic body before deformation. Under the action of a field of volume and surface forces, the elastic body deforms itself and takes a new configuration $\Omega$. The problem is then to determine the displacement field $u$ which associates to the final position $P_{0}$ of each particle of the body before deformation, its position $P$ in the final configuration. 
In order to find a numerical solution to this problem, we used finite elements through a variational formulation. The problem finally reduces to the resolution of a linear system

$$
[\mathbf{K}][\mathbf{U}]=[\mathbf{F}]
$$

where $[\mathbf{K}]$ is the stiffness matrix describing the elastic properties of the solid, [U] corresponds to the unknown displacement field and $[\mathbf{F}]$ represents the external forces. We chose a decomposition of the object volume in a set of tetrahedra, because we already have a triangulation of the outer boundary of the liver. In addition, the geometry of most organs being rather complex, tetrahedra tend to be a good trade-off for such a decomposition.

In addition to the algorithmic complexity, another problem linked with the use of finite elements techniques is due to boundary conditions. In a classical approach, one considers that the deformation is induced by a set of forces applied to the surface of the model and combined with a set of boundary conditions (usually a set of nodes with a null displacement). The force feedback system does not allow the measurement of forces generated by the operator, but only the measurement of the displacement corresponding to the mobile part of the force feedback system. Therefore, we must solve a problem in different format, where our boundary conditions include the displacements of the organ nodes which are in contact with the instrument tip. From this, we have to deduce the global deformation of the system, and the reaction force finally sent back to the operator through the system. (cf. figure 11).

\section{Real time computation of the deformation and forces}

Because of high frequency computations required by force feed-back systems, we have developed a method allowing the computation of both the global model deformation and of the reaction force at a frequency varying between 300 and $500 \mathrm{~Hz}$.

Our algorithm is based on the principles of linearity and superposition of elasticity theory. The superposition principle can be summarised as follows: if we apply to the solid a set of forces creating a displacement field $u$, then this same displacement field can be obtained by applying separately each force, and by adding up their effects. The linearity principle tells us that if a force $f$, applied to the surface of an elastic body creates a displacement $u$, then a force $2 f$ will create a displacement $2 u$.

Consequently, any deformation of our elastic model can be obtained by a linear combination of a set of « elementary » displacements applied to each surface node. An adaptation is required in our case, because displacements are imposed, not forces $[16,17]$. Our method allows the real-time computation of the deformation of a complex organ like the liver under the action of a virtual instrument, along with the reaction force that this organ transmits to the surgical tool (cf. figure 7 , figure 8 and figure 9). 


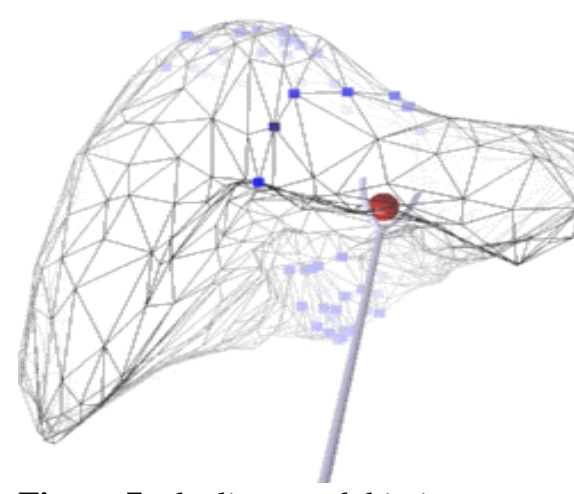

Figure 7: the liver model in its rest shape. The square landmarks correspond to the boundary conditions.

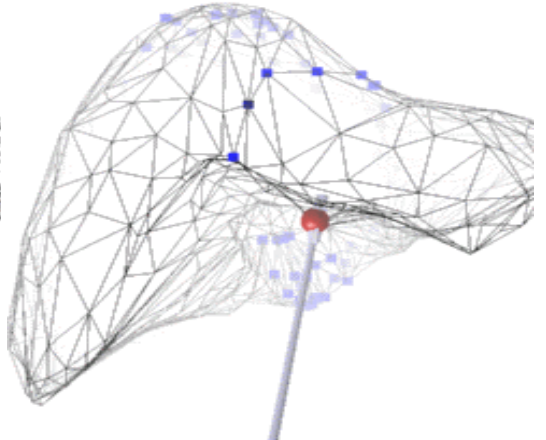

Figure 8: first, the surgical tool comes in contact with the mesh surface. Then, its following motion is converted into a displacement of a set of nodes on the surface.

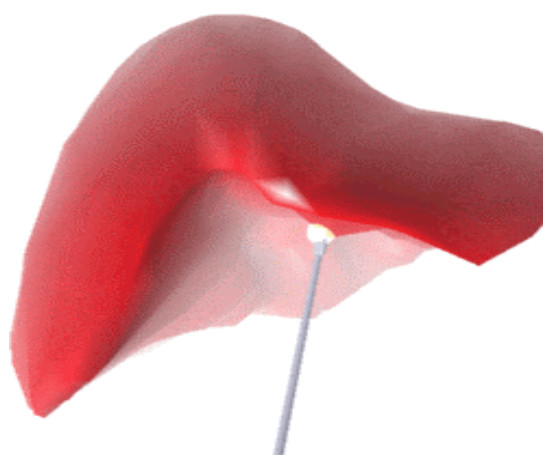

Figure 9: the motion of the surface nodes provides a deformation, computed in real-time. Moreover, a force associated to the deformation is also computed and sent to the force feedback system.

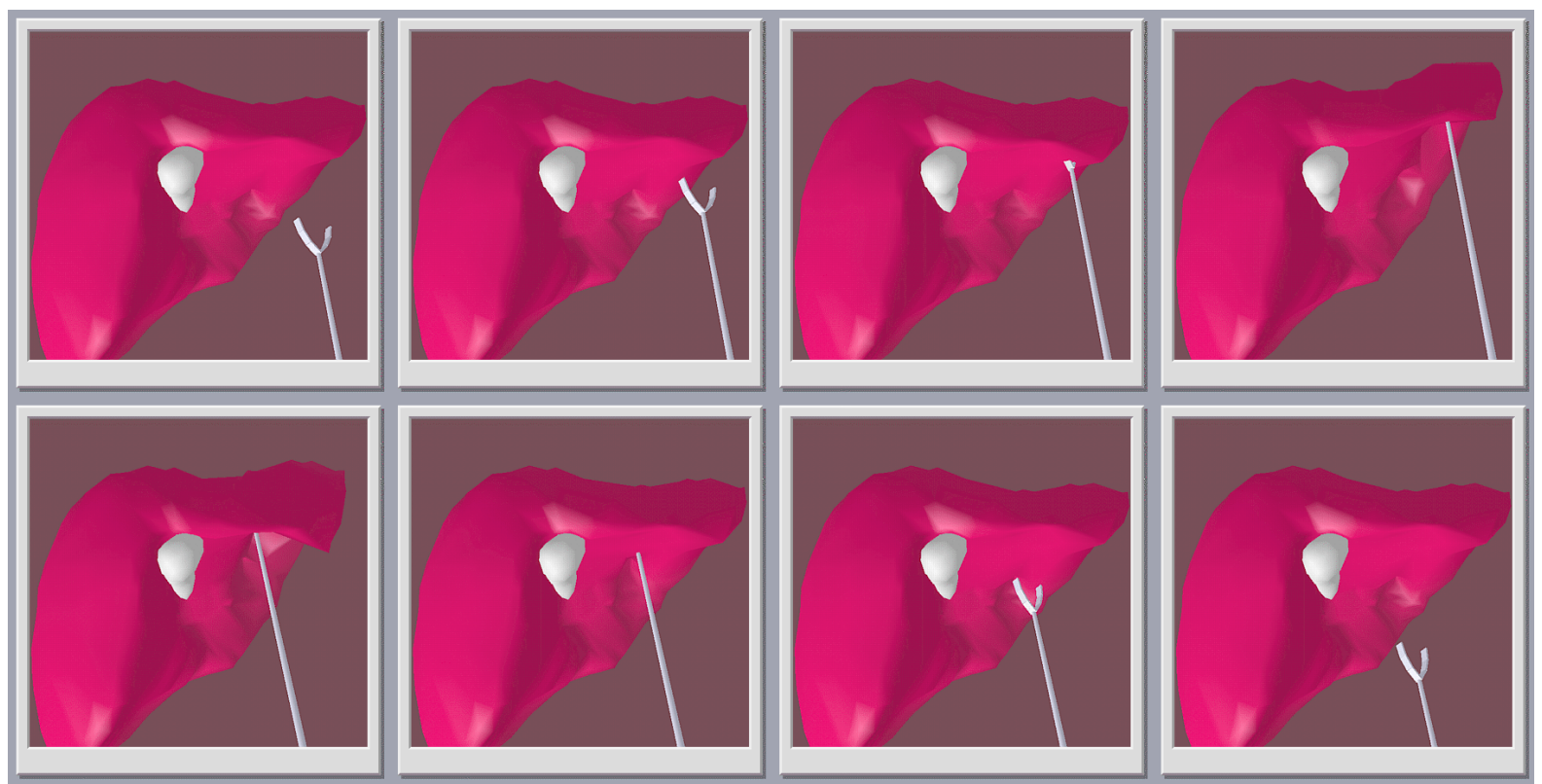

Figure 10: image sequence: real-time interactions with a complex liver model are described. The gall-bladder is also represented. The surgeon feels a force which is correlated with the deformation amplitude and with the geometry of the mesh.

\section{Perspectives}

It is not sufficient to allow the surgeon to manipulate and deform the anatomical model of the liver, we must also ensure that the model behaviour corresponds to anatomical reality. The elastic properties, boundary conditions must be determined for the liver to allow a more realistic behaviour. Visual realism can also be enhanced. We propose the following future improvements:

- nature of hepatic tissues: we attach a elasticity coefficient which depends on the grey-level value of the medical image of the liver. Incidentally, we could take into account the vascular rigidity which plays an important role in the general behaviour of the liver. 
- interactions with other abdominal organs: the liver is not isolated in the abdomen, but it is surrounded by other organs like the gall bladder. Consequently, it is necessary to model these interactions between nearby organs, which could be done by an additional set of boundary conditions.

- texture: a realistic texture, extracted from real video-surgery images could be mapped on the surface of virtual organs to enhance the visual realism.

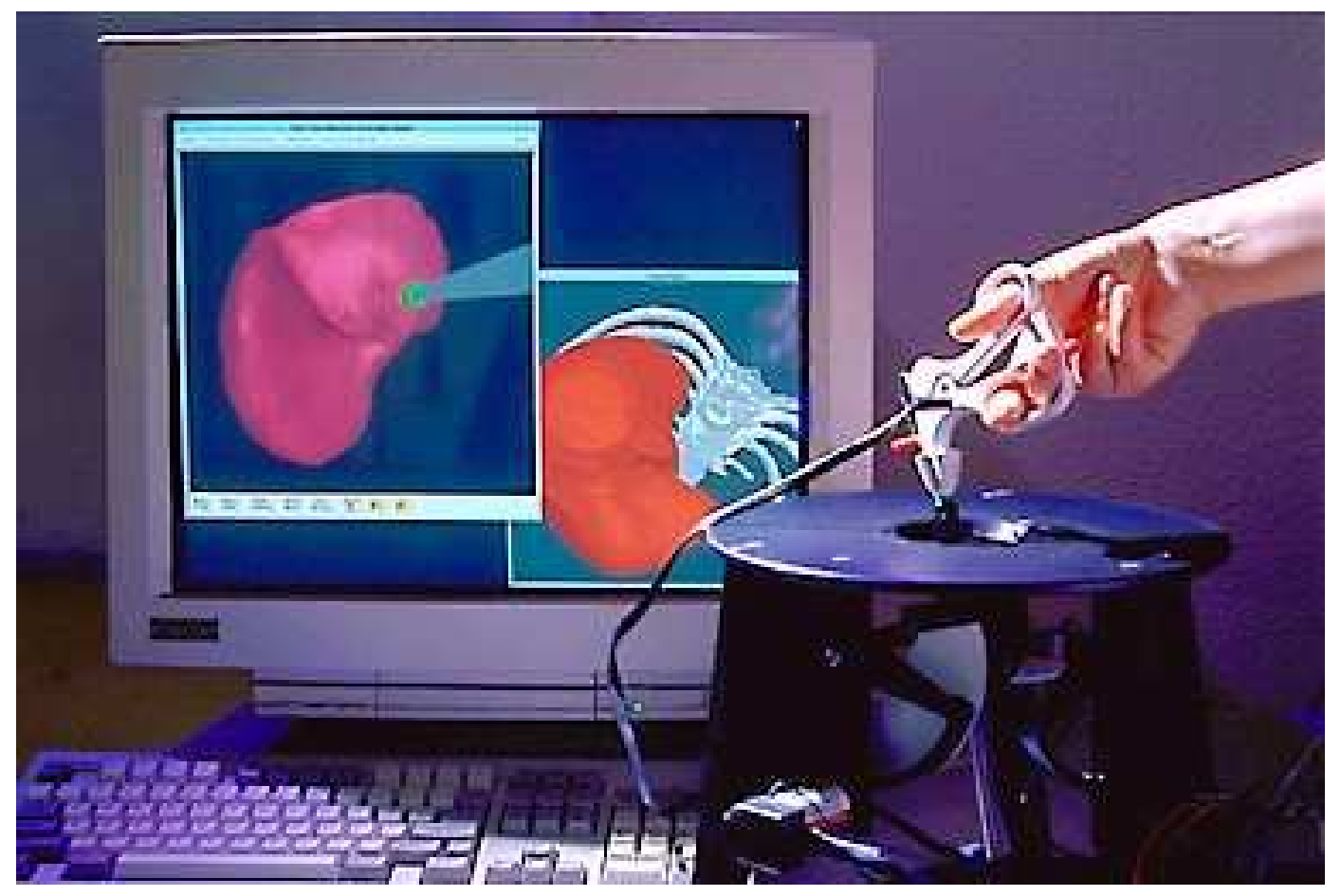

Figure 11: the actual hepatic surgery simulator prototype at INRIA. At the fore-front we can see the force feedback system. The screen shows a scene viewed from the endoscope view point.

\section{Conclusion}

This work must be considered as a first stage towards the building of a practical surgical simulator. We have demonstrated that real time deformation of an elastic model was possible, by combining a physical model with an adequate geometrical representation. We believe that it is essential to have a volumetric modelling of organs, in order to produce realistic deformations, and allow, in the future, the simulation of a realistic hepatectomy. Developing an original and efficient method for the computation of deformations based on linear elasticity, we have been able to simulate real-time interactions with a virtual organ and to compute the contact forces with the surgical instrument. These forces have a physical foundation as they depend on the imposed displacement and are based on linear elasticity. The prototype we have developed allowed for the validation of the algorithms, and a better understanding of the software integration constraints. A new version of this simulator should migrate from INRIA to IRCAD in the near future for further evaluation steps. 


\section{References}

[1] J.-P. Thirion and A. Gourdon. Computing the differential characteristics of iso-intensity surfaces. In CVGIP, pages 190--202, Mars 1995.

[2] H. Delingette, G. Subsol, S. Cotin, and J. Pignon. Simulation de Chirurgie Craniofaciale and Réalité Virtuelle. L'interface des Mondes Réels et Virtuels pages 399-408. Montpellier, France, 1994.

[3] H. Delingette. Simplex Meshes : a General Representation for 3D Shape Reconstruction. Research report \# 2214, INRIA, March 1994.

[4] D. Terzopoulos, J. Platt, A. Barr, and K. Fleisher. Elastically Deformable Models. In Computer Graphics (SIGGRAPH '87), volume 21 No 4, pages 205--214, Juillet 1987.

D. Terzopoulos and K. Fleisher. Modeling Inelastic Deformation : Viscoelasticity, plasticity, fracture. In Computer Graphics (SIGGRAPH '87), volume 22, no. 4, pages 269-278, 1988.

J. C. Platt and A. H. Barr. Constraint Methods for Flexible Models. In Computer Graphics (SIGGRAPH '88), volume 22 No 4, pages 279--288, 1988.

M. Bro-Nielsen. Modelling elasticity in solids using active cubes - application to simulated operations. Computer Vision, Virtual Reality and Robotics in Medecine, volume 905 of Lecture Notes in Computer Science, pages 535-541. Springer, Avril 1995.

[8] K. Waters and D. Terzopoulos. A Physical Model of Facial Tissue and Muscle Articulation. IEEE, 1990.

[9] U.G. Kuehnapfel and B. Neisius. CAD-Based Graphical Computer Simulation in endoscopic surgery. End. Surg. $1: 181-184,1993$.

[10] S. Gibson, J. Samoski, A. Mor, C. Foyck, E. Grimson, T. Kanade, R. Kikinis, H. Lauer and N. McKenzie. Simulating Arthroscopic knee suergery using volumetric object representation, realtime volume rendering and haptic feedback. Proceedings of the first joint conference CVRMedMRCAS' 97, Volume 1205 de Lecture Notes in Computer Science pages 369,378, 1997.

[11] W. Barfield and C. Hendrix. Factors Affecting Presence and Performance in Virtual Environments, Proceedings of MMVR III, Medecine Meets Virtual Reality, chapter 4, pages 2128. IOS Press and Ohmsha, 1995.

[12] S.M. Sukthankar and N.P. Reddy. Force Feedback Issues in Minimally Invasive Surgery, Proceedings of MMVR III, Medecine Meets Virtual Reality, chapter 56, pages 375-379. IOS Press and Ohmsha, 1995.

[13] B.G. Jackson and L.B. Rosenberg. Force Feedback and Medical Simulation. Proceedings of MMVR III, Medecine Meets Virtual Reality, chapitre 24, pages 147-151. IOS Press and Ohmsha, 1995.

[14] Thomas H. Speeter. Three Dimensional Finite Element Analysis of Elastic continua for Tactile Sensing. International Journal of Robotics Research, 11 No 1:1-19, Février 1992.

[15] G. J. Song and N. P. Reddy. Tissue Cutting in Virtual Environment, Proceedings of MMVR'95, chapter 54, pages 359--364. IOS Press and Ohmsha, 1995. 
[16] S. Cotin, H. Delingette and N. Ayache. Real-time Volumetric Deformable Models for Surgery Simulation. Proceedings of VBC' 96, Visualization in Biomedical Computing, volume 1131 of Lecture Notes in Computer Science, pages 535-540. Springer, 1996.

[17] S. Cotin, H. Delingette, J.-M. Clément, V. Tassetti, J. Marescaux and N. Ayache. Geometric and Physical Representations for a Simulator of Hepatic Surgery. Proceedings of MMVR IV, Medecine Meets Virtual Reality, pages 139-151, IOS Press, Janvier 1996. 